\section{Medical students' knowledge and perceptions regarding contraception in Karachi, Pakistan}

Currently home to 180 million people, Pakistan's population continues to rise, with an estimated increase of 66 million in the next 15 years. ${ }^{1}$ While it is encourcountries have shown an interest in limiting their family size and appropriately spacing out pregnancies by means of modern contraceptive methods, most women in developing countries still find it difficult to obtain contraception. Also, failure to correctly and consistently use modern contraceptive methods has led to an increase in the number of unwanted pregnancies. ${ }^{2}$ In Pakistan, the contraceptive prevalence ratio is alarmingly low at $29 \%$. $^{3}$ Consequently physicians need to be able to explain the correct use of different contraceptive methods to patients; however, the only way that this can be achieved is for young doctors to receive proper education about contraception as a component of their lectures on reproductive health while attending medical school.

Medical students gain a basic insight into the pros and cons of various contraceptive methods during their medical training. However, more needs to be done to improve their knowledge about key reproductive and contraceptive issues, especially in Pakistan, a country with a burgeoning population and one of the highest maternal mortality rates. ${ }^{4}$ We conducted a survey to investigate the knowledge and perceptions about contraception of 446 senior medical students (female: male ratio of 11:2) in three medical aging to note that women in developing

schools in Karachi. Figure 1 shows the medical students' awareness of various contraceptive methods.

Students thought that avoiding pregnancy was the key factor in determining the type of contraception used. Students believed the male condom to be the most common (63.7\%) and safest form (41.3\%) of contraception, and also the most effective $(85.4 \%)$ method for preventing the spread of sexually transmitted infections. Many (40\%) students considered the intrauterine device to be the most reliable method, while $12.8 \%$ chose tubal ligation as a reliable method. Coitus interruptus was thought to be the least reliable method.

When asked about the adverse effects of oral contraceptives, $55.2 \%$ of the students cited increased risk of endometrial cancer, $37.4 \%$ thought that oral contraceptives were contraindicated in women with heart disease, and $23.7 \%$ considered menstrual abnormalities to be the most common complication of contraceptive use. Reproductive health is an essential component of the medical student curriculum in Pakistan, and $77 \%$ of the students questioned indicated that university lectures were their source of information.

Needless to say, in order to improve the misperceptions that couples in Pakistan have about contraception it is imperative that doctors possess accurate and comprehensive knowledge about the use and side effects of the available contraceptive methods, since they come into contact with couples who desire smaller families. In our study, gaps were found in medical students' knowledge and perceptions about contraception. Only a small percentage of medical students were able to identify all the problems associated with oral

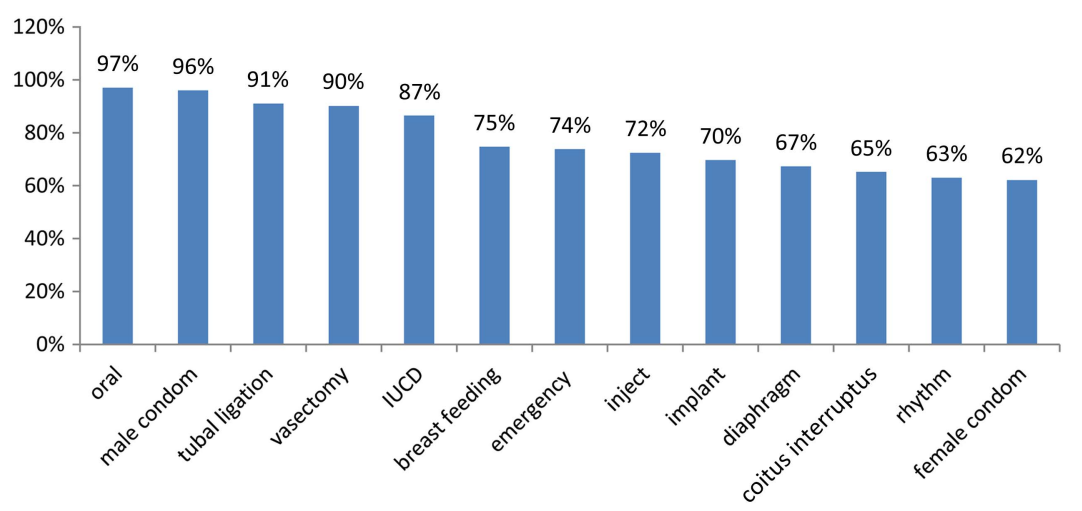

Figure 1 Pakistani medical students' awareness of various contraceptive methods. IUCD, intrauterine contraceptive device. contraceptive use. Medical students need to be familiar with the specific challenges that Pakistan faces regarding contraception including the societal norms that dictate the need for larger families; people's limited understanding of contraception; parents' preference for sons; and the fact that so far as family planning is concerned, women have less authority than their male counterparts..$^{5}$ Looking to the future, appropriately educated physicians will be better able to help their patients understand and appreciate the benefits of improved women's health and smaller family size that result from family planning initiatives.

\section{Muhammad Danish Saleem}

Medical Student, Dow University of Health Sciences, Karachi, Pakistan; mdanishsaleem@gmail.com

\section{Faryal Tahir}

Medical Student, Dow University of Health Sciences, Karachi, Pakistan; Faryal_tahir@hotmail.com

\section{Dania Aijaz Shah*}

Medical Student, Dow University of Health Sciences, Karachi, Pakistan; dania.aijaz.shah@gmail.com

\section{Syed Sami Haider}

Medical Student, Dow University of Health Sciences, Karachi, Pakistan; samihaider31@gmail.com

${ }^{*}$ Corresponding author.

Competing interests None.

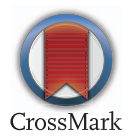

\section{REFERENCES}

1 Population Action International. Pakistan's daunting and deteriorating demographic challenge. http://www.populationaction.org/ blog/2009/04/pakistans-dauntingand-deterior. html [accessed 18 March 2014].

2 Darroch JE, Singh S. Trends in contraceptive need and use in developing countries in 2003, 2008, and 2012: an analysis of national surveys. Lancet 2013;381:1756-1762.

3 Naqvi S, Hashim N, Zareen N, et al. Knowledge, attitude and practice of parous women regarding contraception. J Coll Physicians Surg Pak 2011;21:103-105.

4 Ajmal F, Agha A, Zareen N, et al. Knowledge, attitudes and practices (KAP) regarding sexuality, sexual behaviors and contraceptives among college/university students in Karachi, Pakistan. J Coll Physicians Surg Pak 2011;21:164-168.

5 Mahmood N, Ringheim K. Factors affecting contraceptive use in Pakistan. Pakistan Dev Rev 1996;35:1-22. 